\title{
Efficient Synthesis of a-Oximinoketones using Carboxyl and Nitrite Functionalized Graphene Quantum Dots: Dual role of Nanostructure as a Catalyst and Reagent
}

\author{
Ashkan Shomali ${ }^{1^{*}}$, Hassan Valizadeh ${ }^{2}$ \\ Department of Chemistry, Faculty of Sciences, Azarbaijan Shahid Madani University, P.O. Box 53714-161, \\ Tabriz, Iran. \\ 1. ashkan.shomali@yahoo.com \\ 2. hvalizadeh2@yahoo.com
}

\begin{abstract}
Carboxyl and nitrite functionalized graphene quantum dots (CNGQDs) was used for the efficient synthesis of $\alpha$ oximinoketones under free mineral acid conditions at room temperature. CNGQDs was prepared via o-nitrozation of carboxyl and hydroxyl graphene quantum dots (CHGQDs) and used as a nitrosonium source and also as an efficient acidic catalyst for the synthesis of $\alpha$-oximinoketones. The structure of the catalyst was characterized by FT-IR, XRD, TGA and photoluminescence techniques. The structures of the synthesized products were confirmed by FT-IR, ${ }^{1} \mathrm{H}-\mathrm{NMR}$ and ${ }^{13} \mathrm{C}$-NMR spectroscopic techniques. Stability and recyclability of the prepared homogeneous catalyst was studied in details. Reaction times and yields of the products were compared with previous reported methods resulting high yields and also shorter reaction times.
\end{abstract}

\section{Keywords}

Carboxyl and nitrite functionalized graphene quantum dots; $\alpha$-Oximinoketones; Nitrosation

\section{Academic Discipline And Sub-Disciplines}

Chemistry

\section{SUBJECT CLASSIFICATION}

Organic Chemistry

\section{TYPE (METHOD/APPROACH)}

\author{
NanoCatalyst
}

\section{INTRODUCTION}

a-Oximinoketones are important organic compounds and organic chemists have great interest for the synthesis of these compounds. Significance of these compounds are because of applications of some oximinoketones derivatives in agricultural fields as a herbicides[1-3]. Also a-oximinoketones attached to heterocyclic compounds were used as useful pharmaceuticals[4-6]. Some $\alpha$-oximinoketones derivatives are key intermediates for the synthesis of important compounds like amino acids[7], $\alpha$-diketones[8], nylon[9], nitrosopyrazoles[10]. $\alpha$-Oximinoketones are commonly synthesized via the reaction of carbonyl compounds with hydroxylamine hydrochloride in the presence of sodium nitrite and a mineral acid[11] as a nitrosonium source[12] and used of solid support such as silica gel in the presence of a base[13]. CaO was used as an efficient catalyst for the preparation of oximes at $130^{\circ} \mathrm{C}$ under mild conditions[14]. Recently, we reported TSIL-ONO as a heterogeneous catalyst and effective nitrosonium source for the synthesis of oximinoketones[15]. Although various synthetic methods have been reported for the synthesis of these compounds, however in most of these methods one environmentally harmful mineral acid was used as a catalyst.

Recently graphene quantum dots (GQDs) have received intensive research interest among the chemists because they have important features such as good semiconductivity, high aqueous solubility, biocompatibility, low toxicity, strong photoluminescence and easy functionalization[16-23]. In recent study, we synthesized carboxyl functionalized graphene quantum dots as an acidic nano-catalyst under microwave irradiation for the synthesis of xanthene derivatives[24]. Herein, considering the importance of the $\alpha$-oximinoketones, we reported the use of CNGQDs as an efficient reagent and catalyst for the synthesis of $\alpha$-oximinoketones via the reaction of ketones and diketones under mineral acid free mild and green conditions (Scheme 1). In comparison with reported methods, it was found that the reactions were performed in shorter reaction times with higher yields of products under mild conditions. 
Scheme 1: CNGQDs as a highly effective reagent and nanocatalyst for synthesis of $\alpha$-oximinoketones.

\section{RESULTS AND DISCUSSION}

\section{Preparation and structural features of CNGQDs}

CHGQDs was synthesized according to the literature[24]. The morphology of this compound was studied by TEM image (Figure 1, part A). According to this image uniform distribution $(30-50 \mathrm{~nm})$ of nano-sheets could be seen. CNGQDs was synthesized from freshly prepared CHGQDs according to our recently published paper[25]. The XRD patterns and TGA diagram of CHGQDs and CNGQDs were shown in Figure 1 (part B and C). Interlayer spaces were determined from the XRD patterns as $3.82 \AA$ and $3.77 \AA$ for CHGQDs and CNGQDs respectively. Interlayer spacing for to bulk graphite is 3.34 $\AA$ [26]. Increasing of the interlayer spaces for CHGQDs and CNGQDs in comparison with bulk graphite confirmed the expected modification. Also, comparison of TGA diagrams of CHGQDs and CNGQDs confirmed the interchange of functional group in these compounds. The conversion of $\mathrm{OH}$ groups in CHGQDs to ONO groups in CNGQDs could decrease the hydrogen bond formation between interlayers in CNGQDs causing decomposition of this compound at low temperatures in comparison with CHGQDs.

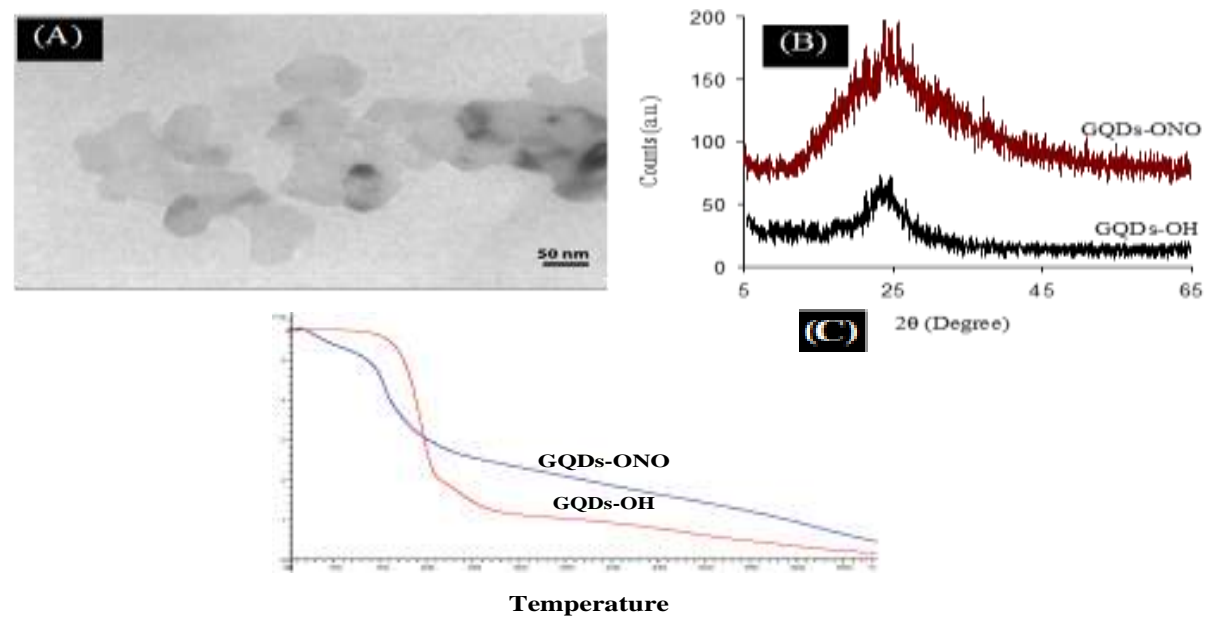

Fig 1: A) TEM image of CNGQDs; B) XRD patterns of CHGQDs and CNGQDs; C) TGA diagram of CHGQDs and CNGQDs.

FT-IR and photoluminescence spectra of CHGQDs and CNGQDs are shown in Figure 2. Absorptions at $1375 \mathrm{~cm}^{-1}$ in CNGQDs spectrum corresponding to $\mathrm{N}=\mathrm{O}$ bond that confirmed modification (Figure 2, part A). The strong photoluminescence peaks at $503 \mathrm{~nm}$ are originated from free zigzag sites with a carbene-like triplet ground state[27]. The fluorescence intensity of CNGQDs is remarkably increased in comparison with CHGQDs approving the successful interconversion of functional groups (Figure $2 \mathrm{~B}$ ). 

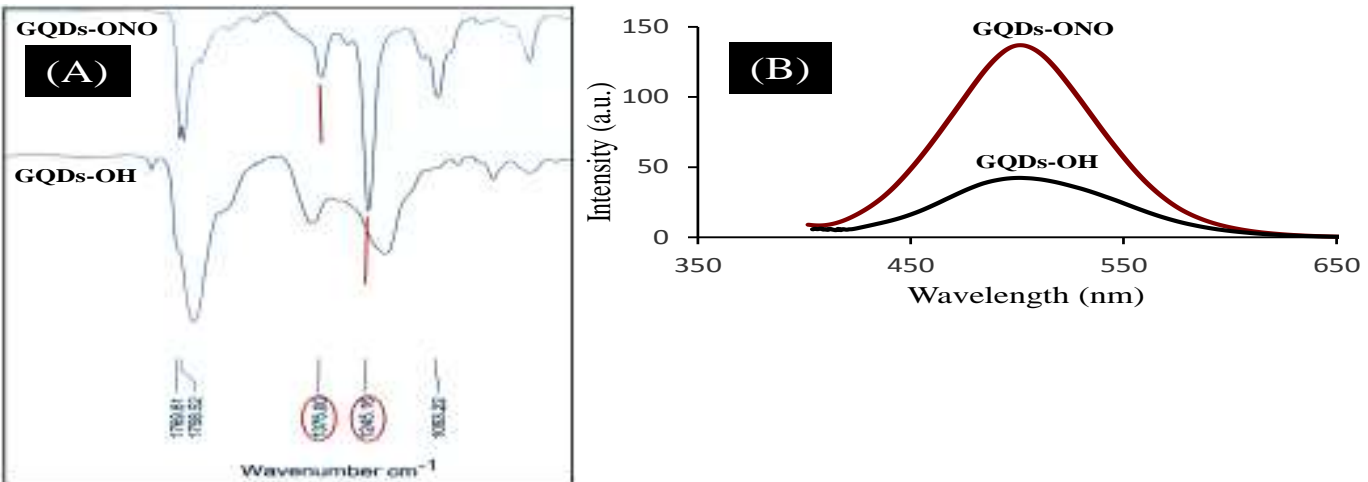

Fig 2: A) FT-IR spectra of CHGQDs and CNGQDs; B) Fluorescence spectra of CHGQDs and CNGQDs.

\section{Reactions}

Malonamide as an activated methylene compound was chosen as a typical carbonyl compound for the oximation reaction in water in the presence of CNGQDs as a nitrosonium source and an acidic catalyst at room temperature. At first, malonamide-2-one-2-oxime (2k) was prepared from the reaction of malonamide $(10 \mathrm{mmol})$ with CNGQDs $(0.2 \mathrm{~g})$ in $50 \mathrm{~min}$ and $65 \%$ yield. For optimization, the reaction was studied with different amounts of catalyst. It was found that the optimum value of catalyst in this reaction is $0.4 \mathrm{~g}$ and $90 \%$ yield of product was achieved under these conditions. The increase of CNGQDs amount had no effect on the reaction efficiency. The participated crude oximinoketone were isolated by filtration under vacuum and then dried at room temperature. For further purification, crude products were recrystallized by water/ethanol. Efficiency of this reagent was compared with reported reagents for the synthesis of oximinoketones under optimized conditions. The results were shown in Table 2. As it can be seen from Table 2, CNGQDs has higher efficiency in comparison. Thus, it can be introduced as an effective catalyst and reagent for the synthesis of oximinoketones from active methylene compounds in the absence of any mineral acid.

Table 1. Synthesis of oximinoketones using nitrite functional graphene quantum dots (CNGQDs).

\begin{tabular}{|c|c|c|c|c|}
\hline Entry & Ketone / Diketone & $\begin{array}{c}\text { Reactant } \\
\text { number }\end{array}$ & Product & $\begin{array}{l}\text { Product } \\
\text { number }\end{array}$ \\
\hline
\end{tabular}


Table 1. (continued)

\begin{tabular}{|c|c|c|c|c|}
\hline Entry & Ketone / Diketone & $\begin{array}{c}\text { Reactant } \\
\text { number }\end{array}$ & Product & $\begin{array}{l}\text { Product } \\
\text { number }\end{array}$ \\
\hline
\end{tabular}


19<smiles>CC1(C)CC(=O)CC(=O)C1</smiles>

20<smiles>O=C1CCCC(=O)C1</smiles>

$1 \mathrm{~s}$<smiles>CC1(C)CC(=O)C(=O)C(=O)C1</smiles>

1t<smiles>O=C1CCCC(=O)C1=O</smiles>

2s

2t

Table 2. Comparison of the yields of products with reported values.

\begin{tabular}{|c|c|c|c|c|c|c|}
\hline \multirow[b]{2}{*}{ Entry } & \multirow{2}{*}{$\begin{array}{l}\text { Product } \\
\text { number }\end{array}$} & \multirow{2}{*}{$\begin{array}{l}\text { Time } \\
(\mathrm{min})\end{array}$} & \multicolumn{2}{|c|}{ M.P ( C) } & \multicolumn{2}{|c|}{ Yield $^{\mathrm{a}}(\%)$} \\
\hline & & & Found & $\begin{array}{c}\text { Reported } \\
\text { [Ref.] }\end{array}$ & Found & $\begin{array}{c}\text { Reported } \\
\text { [Ref.] }\end{array}$ \\
\hline 1 & $2 a$ & 25 & $128-129$ & $128-130$ [28] & 90 & 90 [28] \\
\hline 2 & $2 b$ & 30 & - & - & 80 & 65 [15] \\
\hline 3 & 2c & 30 & $149-150$ & $148-150[28]$ & 98 & 93 [28] \\
\hline 4 & $2 d$ & 25 & $140-141$ & $141-143[28]$ & 98 & 93 [28] \\
\hline 5 & $2 e$ & 30 & $162-163$ & $162-163$ [29] & 92 & 87 [29] \\
\hline 6 & $2 f$ & 30 & $125-126$ & $126-127$ [29] & 89 & 68 [29] \\
\hline 7 & $2 g$ & 20 & $115-116$ & $114-115[29]$ & 86 & 77 [29] \\
\hline 8 & $2 \mathrm{~h}$ & 25 & - & - & 95 & 83 [30] \\
\hline 9 & $2 \mathbf{i}$ & 20 & $145-146$ & $145-149[15]$ & 90 & 75 [12] \\
\hline 10 & $2 j$ & 20 & $130-131$ & $129-131$ [15] & 89 & 86 [12] \\
\hline 11 & $2 k$ & 30 & $167-168$ & $168-170$ [15] & 98 & 95 [15] \\
\hline 12 & 21 & 30 & $129-130$ & $129-132$ [15] & 98 & 94 [15] \\
\hline 13 & $2 m$ & 30 & - & - & 90 & 85 [15] \\
\hline 14 & $2 n$ & 30 & - & - & 91 & 89 [15] \\
\hline 15 & 20 & 25 & - & - & 85 & 85 [15] \\
\hline 16 & $2 p$ & 30 & - & - & 96 & 84 [12] \\
\hline 17 & $2 q$ & 25 & - & - & 82 & $61[30]$ \\
\hline 18 & $2 r$ & 25 & - & - & 87 & 75 [15] \\
\hline 19 & $2 s$ & 30 & - & - & 81 & 60 [30] \\
\hline 20 & $2 t$ & 30 & - & - & 89 & 70 [30] \\
\hline
\end{tabular}

${ }^{a}$ Isolated yield.

\section{CONCLUSION}

In summary, we introduced a new efficient reagent with dual role (as nitrosonium source and acid catalyst) for the oximination of active methylene compounds. Mild reaction conditions in water as a green solvent and simple workup procedure are the advantages of this method. Excellent yields of the products were obtained in shorter reaction times. 


\section{EXPERIMENTAL}

\section{Materials and instrumentation}

All reagents were purchased from Merck Company and used without further purification. Infrared spectra were recorded with $\mathrm{KBr}$ on a Perkin-Elmer FT-IR spectrometer. Melting points were determined in open glass-capillaries using a Stuart melting point apparatus. ${ }^{1} \mathrm{H}$ and ${ }^{13} \mathrm{C}$ NMR spectra were recorded on a Bruker Avance AC-400MHz using DMSO- $\mathrm{d}_{6}$ as the deuterated solvents and TMS as an internal standard. Elemental analysis was determined by CHNSO; Euro EA; model EA3000. CNGQDs structure was characterized by X-ray diffraction (XRD) (Bruker AXS model D8 Advance). Transmission-electron microscopy (TEM) was recorded on a Philips CM-10 (Eindhoven, The Netherlands). Fluorescence spectra and intensity measurements were carried out using an FP-6200 spectrofluorometer (JASCO Corporation, Tokyo, Japan). Thermal gravimetric analysis (TGA) was determined using Mettler Toledo TGA-DTA STAR SW.8.10.

\section{Synthesis of CNGQDs}

CNGQDs was synthesized from carboxyl and hydroxyl functionalized graphene quantum dots (CHGQDs) according to the reported method in our recently published work[25]. The solution of sodium nitrite in water (18 $\mathrm{mL}$ ) was added slowly to the freshly prepared CHGQDs aqueous solution ( $25 \mathrm{~g} \mathrm{CHGQDs}$ was dissolved in water $100 \mathrm{~mL}$ ) at $0-5{ }^{\circ} \mathrm{C}$ in $30 \mathrm{~min}$. After saturating the prepared solution by $\mathrm{NaCl}(10 \mathrm{~g})$, the modified GQDs were extracted with ethyl acetate $(3 \times 25 \mathrm{~mL})$. The ethyl acetate was removed under reduced pressure at $40{ }^{\circ} \mathrm{C}$ and the residue was dried under vacuum at room temperature to produce CNGQDs (19.5 g) (Scheme 2).

\section{Scheme 2: Preparation of CNGQDs from CHGQDs.}

\section{General procedure for the synthesis of $\alpha$-oximinoketones (2a-t)}

Diketone or ketone $(10 \mathrm{mmol})$ were dissolved in water or mixed water - alcohol $(12 \mathrm{~mL})$ and was stirred at room temperature. Then, CNGQDs $(0.4 \mathrm{~g})$ was added slowly while stirring in $20 \mathrm{~min}$. The progress of the reaction was monitored by thin layer chromatography (TLC) until the products were appeared as precipitated. In order to separate catalyst and unreacted ingredients from products, the precipitated were filtered and washed three times with cold water. The precipitated crude products were further purified by recrystallization (water/ethanol) to afford the pure products (2a-t) in high yields (Table 2). In order to isolate the catalyst, the separated aqueous solution was extracted by ethyl acetate in three times. Finally, the catalyst was revived from aqueous solution using vacuum and temperature (rotary evaporator). Recyclability of the catalyst was proved by repeating the sample reaction 5 times and the results was represented in Figure 3. Regardless decrease of yields proves appropriate recyclability of the catalyst in sample reaction.

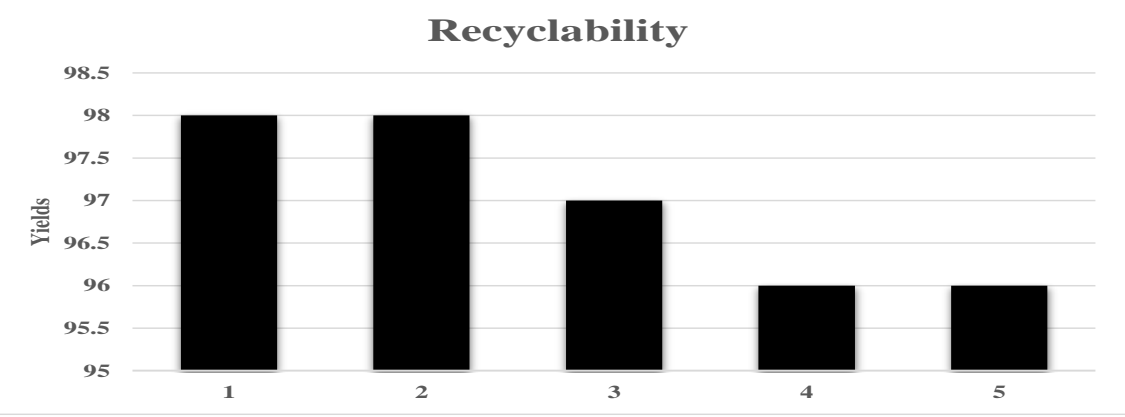

Fig 3: Recyclability study diagram of separated catalyst.

\section{Selected spectroscopic data}

\section{1-Phenyl-1,2-ethanedione-2-oxime (2a)}

Yield 90\%; mp 128-129 ${ }^{\circ} \mathrm{C}$ (literature[24] 128-130 ${ }^{\circ} \mathrm{C}$ ); ${ }^{1} \mathrm{H}$ NMR (DMSO- $d_{6}, 400 \mathrm{MHz}$ ) $\delta: 7.73-7.77$ (m, 2H, ph-H), 7.81$7.85(\mathrm{~m}, 3 \mathrm{H}, \mathrm{ph}-\mathrm{H}), 8.12(\mathrm{~s}, 1 \mathrm{H},=\mathrm{CH}), 12.75(\mathrm{~s}, 1 \mathrm{H}, \mathrm{OH}) .{ }^{13} \mathrm{C}$ NMR $\left(\mathrm{DMSO}-d_{6}, 150 \mathrm{MHz}\right) \delta: 112.32,117.65,125.63$ 
128.45, 148.63, 161.54. IR (KBr) U $U_{\max }: 3421(\mathrm{br}, \mathrm{OH}), 1688,1685,1614,1450,1318 \mathrm{~cm}^{-1}$. Anal. Calcd. for $\mathrm{C}_{8} \mathrm{H}_{7} \mathrm{NO}_{2}(\%):$ C, 64.42; H, 4.73; N, 9.39. Found: C, 64.87; H, 4.71; N, 9.35.

\section{1-(2-Hydroxyphenyl)-1,2-ethanedione-2-oxime (2b)}

Yield 80\%;[13] ${ }^{1} \mathrm{H}$ NMR (DMSO- $\left.d_{6}, 400 \mathrm{MHz}\right) \delta: 6.35$ (br, 1H, OH), 7.41-7.44 (m, 1H, Ph-H), 7.60-7.65 (m, 1H, ph-H), 7.75-7.78 (m, 2H, ph-H), $8.19(\mathrm{~s}, 1 \mathrm{H},=\mathrm{CH}), 12.82(\mathrm{~s}, 1 \mathrm{H}, \mathrm{OH}) .{ }^{13} \mathrm{C}$ NMR (DMSO-d, $\left.150 \mathrm{MHz}\right) \delta: 114.32,117.56,119.75$ 121.32, 122.52, 122.98, 149.96, 165.85. IR (KBr) U max: 3449 (br, OH), 1684, 1531, $1384 \mathrm{~cm}^{-1}$. Anal. Calcd. for $\mathrm{C}_{8} \mathrm{H}_{7} \mathrm{NO}_{3}$ (\%): C, 58.18; H, 4.27; N, 8.48. Found: C, 59.08; H, 4.26; N, 8.46.

\section{1,3-Diphenyl-1,2,3,-propanetrione-2-oxime (2i)}

Yield \%; mp 145-146 ${ }^{\circ} \mathrm{C}$ (literature[13] 145-149 ${ }^{\circ} \mathrm{C}$ ); ${ }^{1} \mathrm{H}$ NMR (DMSO- $d_{6}, 400 \mathrm{MHz}$ ) $\delta: 7.61-7.68(\mathrm{~m}, 4 \mathrm{H}, \mathrm{ph}-\mathrm{H}), 7.75-7.79$ $(\mathrm{m}, 2 \mathrm{H}, \mathrm{ph}-\mathrm{H}), 7.81-7.85(\mathrm{~m}, 4 \mathrm{H}, \mathrm{ph}-\mathrm{H}), 12.87(\mathrm{~s}, 1 \mathrm{H}, \mathrm{OH}) .{ }^{13} \mathrm{C}$ NMR (DMSO- $\left.d_{6}, 150 \mathrm{MHz}\right) \delta: 109.78,112.25,127.12$, 144.80, 158.14, 160.21. IR (KBr) U max: $3383(\mathrm{br}, \mathrm{OH}), 1701,1685,1590,1458,1370 \mathrm{~cm}^{-1}$. Anal. Calcd. for $\mathrm{C}_{15} \mathrm{H}_{11} \mathrm{NO}_{3}$ (\%): C, 71.14; H, 4.38; N, 5.53. Found: C, 71.23; H, 4.39; N, 5.52.

\section{1-Phenyl-1,2,3,-butanetrione-2-oxime (2j)}

Yield 89\%; mp 130-131 ${ }^{\circ} \mathrm{C}$ (literature[13] 129-131 $\left.{ }^{\circ} \mathrm{C}\right) ;{ }^{1} \mathrm{H}$ NMR (DMSO- $\left.d_{6}, 400 \mathrm{MHz}\right) \delta: 2.51(\mathrm{~s}, 3 \mathrm{H}, \mathrm{Me}), 7.56-7.60(\mathrm{~m}$, $2 \mathrm{H}, \mathrm{Ph}-\mathrm{H}), 7.71-7.75(\mathrm{~m}, 1 \mathrm{H}, \mathrm{ph}-\mathrm{H}), 7.77-7.80(\mathrm{~m}, 2 \mathrm{H}, \mathrm{ph}-\mathrm{H}), 13.04(\mathrm{~s}, 1 \mathrm{H}, \mathrm{OH}) .{ }^{13} \mathrm{C}$ NMR (DMSO-d, $\left.150 \mathrm{MHz}\right) \delta: 19.73$, 114.05, 124.16, 128.74, 130.00, 141.10, 154.87, 162.36. IR (KBr) $U_{\max }: 3379$ (br, OH), 1689, 1579, $1451,1372 \mathrm{~cm}^{-1}$. Anal. Calcd. for $\mathrm{C}_{10} \mathrm{H}_{9} \mathrm{NO}_{3}(\%)$ : C, 62.82; $\mathrm{H}, 4.74 ; \mathrm{N}, 7.33$. Found: $\mathrm{C}, 63.02 ; \mathrm{H}, 4.75 ; \mathrm{N}, 7.31$.

\section{Malonamide-2-one-2-oxime (2k)}

Yield 98\%; mp 167-168 ${ }^{\circ} \mathrm{C}$ (literature[13] 168-170 ${ }^{\circ} \mathrm{C}$ ); ${ }^{1} \mathrm{H}$ NMR (DMSO- $\left.d_{6}, 400 \mathrm{MHz}\right) \delta$ : 7.73-7.79 (br, 2H, NH $\left.\mathrm{H}_{2}\right), 7.32-7.36$ (br, 2H, NH $\mathrm{H}_{2}, 12.04(\mathrm{~s}, 1 \mathrm{H}, \mathrm{OH}) .{ }^{13} \mathrm{C}$ NMR (DMSO-d, $\left.\mathrm{MHz}\right) \delta: 138.63,159.32,162.52 . \mathrm{IR}(\mathrm{KBr}) \mathrm{U}_{\max }: 3431(\mathrm{br}, \mathrm{OH}), 3310$ $(\mathrm{N}-\mathrm{H}), 3211(\mathrm{~N}-\mathrm{H}), 1680,1675,1437,1261 \mathrm{~cm}^{-1}$. Anal. Calcd. for $\mathrm{C}_{3} \mathrm{H}_{5} \mathrm{~N}_{3} \mathrm{O}_{3}(\%)$ : C, 27.49; H, 3.84; N, 32.05. Found: C, $28.14 ; \mathrm{H}, 3.85 ; \mathrm{N}, 31.58$.

\section{Methyl 4-methoxy-2,3-dione-2-oxime butanoate (2I)}

Yield 98\%; mp 129-132 ${ }^{\circ} \mathrm{C}$ (literature[13] 129-132 ${ }^{\circ} \mathrm{C}$ ); ${ }^{1} \mathrm{H}$ NMR (DMSO- $\left.d_{6}, 400 \mathrm{MHz}\right) \delta: 3.25\left(\mathrm{~s}, 3 \mathrm{H}, \mathrm{OCH}_{3}\right), 3.68(\mathrm{~s}, 3 \mathrm{H}$, $\left.\mathrm{OCH}_{3}\right), 12.68(\mathrm{~s}, 1 \mathrm{H}, \mathrm{OH}) .{ }^{3} \mathrm{C}$ NMR (DMSO- $\left.d_{6}, 150 \mathrm{MHz}\right) \delta: 59.65,60.25,135.25,161.87,163.45 . \mathrm{R}(\mathrm{KBr}) \mathrm{U}_{\max }: 3330$ (br, $\mathrm{OH}), 1743,1691,1627,1437,1388 \mathrm{~cm}^{-1}$. Anal. Calcd. for $\mathrm{C}_{6} \mathrm{H}_{9} \mathrm{NO}_{5}$ (\%): C, 41.15; H, 5.18; N, 8.00. Found: C, 42.10; H, 5.16; N, 7.98 .

\section{Benzyl 2,3-dione-2-oxime butanoate (2m)}

Yield 90\%;[13] ${ }^{1} \mathrm{H}$ NMR (DMSO- $\left.d_{6}, 400 \mathrm{MHz}\right) \delta: 2.50$ (s, 3H, Me), 3.95 (s, 2H, OCH OCH , 7.52-7.58 (m, 2H, ph-H), 7.67-7.72 $(\mathrm{m}, 1 \mathrm{H}, \mathrm{ph}-\mathrm{H}), 7.75-7.78(\mathrm{~m}, 2 \mathrm{H}, \mathrm{ph}-\mathrm{H}), 13.08(\mathrm{~s}, 1 \mathrm{H}, \mathrm{OH}) .{ }^{13} \mathrm{C}$ NMR (DMSO-d, $\left.150 \mathrm{MHz}\right) \delta: 36.78,71.41,113.63$, 117.87, 119.56, 122.36, 149.79, 160.52, 163.53. IR (KBr) $U_{\max }: 3331$ (br, OH), 1746, 1698, 1627, $1498,1379 \mathrm{~cm}^{-1}$. Anal. Calcd. for $\mathrm{C}_{11} \mathrm{H}_{11} \mathrm{NO}_{4}(\%): \mathrm{C}, 59.73 ; \mathrm{H}, 5.01 ; \mathrm{N}, 6.33$. Found: $\mathrm{C}, 60.23 ; \mathrm{H}, 4.99 ; \mathrm{N}, 6.32$.

\section{Methyl 2,3-dione-2-oxime butanoate (2n)}

Yield 85\%;[13] ${ }^{1} \mathrm{H}$ NMR (DMSO- $\left.d_{6}, 400 \mathrm{MHz}\right) \delta: 2.35$ (s, 3H, $\left.\mathrm{CH}_{3}\right), 3.78$ (s, 3H, $\left.\mathrm{OCH}_{3}\right), 13.34(\mathrm{~s}, 1 \mathrm{H}, \mathrm{OH}) .{ }^{13} \mathrm{C} \mathrm{NMR}$ (DMSO-d $\left.d_{6}, 150 \mathrm{MHz}\right) \delta: 31.25,62.53,147.69,161.53,165.82$. IR (KBr) U max: $3448(\mathrm{br}, \mathrm{OH}), 1751,1654,1452,1373 \mathrm{~cm}^{-1}$ Anal. Calcd. for $\mathrm{C}_{5} \mathrm{H}_{7} \mathrm{NO}_{4}(\%)$ : C, 41.38; $\mathrm{H}, 4.86 ; \mathrm{N}, 9.65$. Found: C, 41.42; H, 4.88; N, 9.63 .

\section{Methyl 2,3-dione-2-oxime hexanoate (20)}

Yield 91\%;[13] ${ }^{1} \mathrm{H}$ NMR (DMSO- $d_{6}, 400 \mathrm{MHz}$ ) $\delta: 1.02-1.09$ (t, 3H, $\left.\mathrm{CH}_{3}\right), 1.15-1.17\left(\mathrm{~m}, 2 \mathrm{H}, \mathrm{CH}_{2}\right), 2.61-2.66\left(\mathrm{t}, 2 \mathrm{H}, \mathrm{CH}_{2}\right)$ $3.65\left(\mathrm{~s}, 3 \mathrm{H}, \mathrm{OCH}_{3}\right), 12.89(\mathrm{~s}, 1 \mathrm{H}, \mathrm{OH}) .{ }^{13} \mathrm{C} \mathrm{NMR}\left(\mathrm{DMSO}-d_{6}, 150 \mathrm{MHz}\right) \delta: 15.52,18.45,23.36,62.52,145.56,158.89$, 164.75. IR (KBr) Umax: $3354(\mathrm{br}, \mathrm{OH}), 1726,1694,1627,1458,1374 \mathrm{~cm}^{-1}$. Anal. Calcd. for $\mathrm{C}_{7} \mathrm{H}_{11} \mathrm{NO}_{4}(\%): \mathrm{C}, 48.55 ; \mathrm{H}$, $6.40 ; \mathrm{N}, 8.09$. Found: C, 48.74; H, 6.43; N, 8.08.

\section{2,4-Dione-3-oxime pentane (2q)}

Yield 82\%;[26] ${ }^{1} \mathrm{H}$ NMR (DMSO- $\left.d_{6}, 400 \mathrm{MHz}\right) \delta: 2.15\left(\mathrm{~s}, 3 \mathrm{H}, \mathrm{CH}_{3}\right), 2.37\left(\mathrm{~s}, 3 \mathrm{H}, \mathrm{CH}_{3}\right), 13.04(\mathrm{~s}, 1 \mathrm{H}, \mathrm{OH}) .{ }^{13} \mathrm{C} \mathrm{NMR}$ (DMSO-d $\left.d_{6}, 150 \mathrm{MHz}\right) \delta: 31.25,29.81,137.79,159.51,161.72$. IR (KBr) U $U_{\max }: 3437(\mathrm{br}, \mathrm{OH}), 1747,1634,1425,1351 \mathrm{~cm}^{-1}$. Anal. Calcd. for $\mathrm{C}_{5} \mathrm{H}_{7} \mathrm{NO}_{3}$ (\%): C, 46.51; $\mathrm{H}, 5.46$; N 10.85. Found: C, 46.53; $\mathrm{H}, 5.47 ; \mathrm{N}, 10.83$.

\section{3,5-Dione-4-oxime heptane (2r)}

Yield 87\%;[15] ${ }^{1} \mathrm{H}$ NMR (DMSO- $\left.d_{6}, 400 \mathrm{MHz}\right) \delta: 1.24\left(\mathrm{t}, 3 \mathrm{H}, \mathrm{CH}_{3}\right), 1.14\left(\mathrm{t}, 3 \mathrm{H}, \mathrm{CH}_{3}\right), 2.24\left(\mathrm{~m}, 2 \mathrm{H}, \mathrm{CH}_{2}\right), 2.18\left(\mathrm{~m}, 2 \mathrm{H}, \mathrm{CH}_{2}\right)$, $13.02(\mathrm{~s}, 1 \mathrm{H}, \mathrm{OH}) .{ }^{13} \mathrm{C}$ NMR (DMSO-d $\left.d_{6}, 150 \mathrm{MHz}\right) \delta: 18.28,19.72,30.16,32.42,136.70,159.43,160.63$. IR (KBr) Umax: 3435 (br, OH), 1748, 1641, 1430, $1356 \mathrm{~cm}^{-1}$. Anal. Calcd. for $\mathrm{C}_{5} \mathrm{H}_{11} \mathrm{NO}_{3}(\%): \mathrm{C}, 53.49 ; \mathrm{H}, 7.05 ; \mathrm{N}, 8.91$. Found: C, 53.50; $\mathrm{H}, 7.06 ; \mathrm{N}, 8.89$. 


\section{ACKNOWLEDGMENTS}

The partial financial assistance from the Research Vice Chancellor of Azarbaijan Shahid Madani University is gratefully acknowledged.

\section{REFERENCES}

[1] Lauer, M., Zipperer, B. and Goetz, N. Eur. Pat. Appl. Ep. 1991, 77, 409.

[2] Benoit, R., Sauter, H. and Kirstgen, R. Eur. Pat. Appl. Ep. 1992, 188, 498.

[3] Misslitz, U., Meyer, N. and Kast, J. Ger. Offen. DE 4. 1991, 18, 623.

[4] Mohan, R. R. 1991. Synthesis of thiazolidinyl-benzylidene/methyl benzylidene-hydrazides and their Mannich bases as CNS active and anti-inflammatory agents. Indian Drugs. 29 (1991), 120-122.

[5] Plate, R. Eur. Pat. Appl. Ep. 1993, 279, 559.

[6] Lazarevski, G. and Djokic, S. Eur. Pat. Appl. Ep. 1991, 35, 448.

[7] McOmie, J. F. W. 1973. Protective Groups in Organic Chemistry, vol. 46, Plenum Press, London, New York.

[8] Huang, L., Cheng, K., Yao, B., Xie, Y. and Zhang, Y. 2011. Iron-promoted C-C bond cleavage of 1,3-diketones: A route to 1,2-diketones under mild reaction conditions. J. Org. Chem. 76 (Jun, 2011), 5732-5737.

[9] Weissermer, K. and Arpe, H. J. 1978. Industrial Organic Chemistry, Springer Verlag, p.222.

[10] Cameron, M., Gowenlock, B. G. and Boyed, A. S. F. 1996. Studies in nitrosopyrazoles. Part 1. Preparative and spectroscopic studies of some 3,5-dialkyl-4-nitrosopyrazoles. J. Chem. Soc. Perkin. Trans. 2. (1996), 2271-2274.

[11] Ren, R. X. and Ou, W. 2001. Preparation of cyclic ketoximes using aqueous hydroxylamine in ionic liquids. Tetrahedron Lett. 42 (Nov, 2001), 8445-8446.

[12] Zolfigol, M. A. 2001. An efficient and chemoselective method for oximination of $\beta$-diketones under mild and heterogeneous conditions. Molecules. 6 (Jul, 2001), 694-698.

[13] Hajipour, A. R., Mohammadpoor-Baltrok, I., Nikbaghat, K. and Imanzadeh, G. 1999. Solid-phase synthesis of oximes. Synth. Comm. 29 (May, 1999), 1697-1701.

[14] Sharghi, H. and Sarvari, M. H. 2000. A mild and versatile method for the preparation of oximes by use of calcium oxide 1. J. Chem. Research (S). (Oct, 2000), 24-25.

[15] Valizadeh, H., Shomali, A. and Gholipour, H. 2012. Nitrite ionic liquids (IL-ONO and [bmim] $\mathrm{NO}_{2}$ ) as effective nitrosonium sources for the synthesis of a-oximinoketones under mild heterogeneous conditions. Chin. J. Chem. 30 (Jul, 2012), 163-166.

[16] Welsher, K., Liu, Z., Daranciang, D. and Dai, H. 2008. Selective probing and imaging of cells with single walled carbon nanotubes as near-infrared fluorescent molecules. Nano Lett. 8 (Jan, 2008), 586-590.

[17] (a) Liu, R., Wu, D., Liu, S., Koynov, K., Knoll, W. and Li, Q. 2009. An aqueous route to multicolor photoluminescent carbon dots using silica spheres as carriers. Angew. Chem. 121 (2009), 4668. (b) Liu, R.; Wu, D.; Liu, S.; Koynov, K.; Knoll, W.; Li, Q. 2009. An Aaueous route to multicolor photoluminescent carbon dots using silica spheres as carriers. Angew. Chem. Int. Ed. 48 (Apr, 2009), 4598-4601.

[18] Zhu, H., Wang, X., Li, Y., Wang, Z., Yang, F. and Yang, X. 2009. Microwave synthesis of fluorescent carbon nanoparticles with electrochemiluminescence properties. Chem. Commun. (Jul, 2009), 5118-5120.

[19] Yang, S. T., Wang, X., Wang, H. F., Lu, F. S., Luo, P. J. G., Cao, L., Meziani, M. J., Liu, J. H., Liu, Y., Chen, M., Huang, Y. and Sun, Y. P. 2009. Carbon dots as nontoxic and high-performance fluorescence imaging agents. J. Phys. Chem. C. 113 (Sept, 2009), 18110-18114.

[20] Zhao, Q. L., Zhang, Z. L., Huang, B. H., Peng, J., Zhang, M. and Pang, D. W. 2008. Facile preparation of low cytotoxicity fluorescent carbon nanocrystals by electrooxidation of graphite. Chem. Commun. (Sept, 2008), 51165118.

[21] Cao, L., Wang, X., Meziani, M. J., Lu, F., Wang, H., Luo, P. G., Lin, Y., Harruff, B. A., Veca, L. M., Murray, D., Xie, S. Y. and Sun, Y. P. 2007. Carbon dots for multiphoton bioimaging. J. Am. Chem. Soc. 129 (Aug, 2007), 11318-11319.

[22] Zheng, L. Y., Chi, Y. W., Dong, Y. Q., Lin, J. P. and Wang, B. B. 2009. Electrochemiluminescence of eater-soluble carbon nanocrystals released slectrochemically from Graphite. J. Am. Chem. Soc. 131 (Mar, 2009), 4564-4565.

[23] Pan, D., Guo, L., Zhang, J., Xi, C., Xue, Q. H., Huang, J. and et al. 2012. Cutting sp ${ }^{2}$ clusters in graphene sheets into colloidal graphene quantum dots with strong green fluorescence. J. Mater. Chem. 22 (Jan, 2012), 3314-3318.

[24] Shomali, A., Valizadeh, H., Banan, A. and Mohammad-Rezaei, R. 2015. Efficient synthesis of Xanthene derivatives using carboxyl functionalized graphene quantum dots as an acidic nano-catalyst under microwave irradiation. RSC Advances. 5 (Oct, 2015), 88202-88208. 
[25] Valizadeh, H., Shomali, A., Nourshargh, S. and Mohammad-Rezaei, R. 2015. Carboxyl and nitrite functionalized graphene quantum dots as a highly active reagent and catalyst for rapid diazotization reaction and synthesis of azo-dyes under solventfree conditions. Dyes and Pigments. 113 (Sept, 2015), 522-528.

[26] Kelly, K. F. and Billups, W. E. 2013. Synthesis of Soluble Graphite and Graphene. Accounts of Chemical Research. 46 (Nov, 2013), 4-13.

[27] Pan, D., Zhang, J., Li, Z. and Wu, M. 2010. Hydrothermal route for cutting graphene sheets into blue-luminescent graphene quantum dots. Adv Mater. 22 (Feb, 2010), 734-738.

[28] Mohammed, A. H. A. and Nagendrappa, G. 2003. A remarkably simple -oximation of ketones to 1,2-dione monooximes using the chlorotrimethylsilane-isoamyl nitrite combination. Tetrahedron Lett. 44 (Mar, 2003), 27532755.

[29] Ferris, A. 1959. Communications- a-oximino ketones. I. The "normal" and "abnormal" beckmann rearrangements. J. Org. Chem. 24 (Apr, 1959), 580-581.

[30] Zolfigol, M. A. and Choghamarani, A. G. 2003. Silica sulfuric acid/ $\mathrm{NaNO}_{2}$ as a novel heterogeneous system for the chemoselective $\alpha$-nitrosation of $\beta$-diketones under mild conditions. Phosphorus, Sulfur, and Silicon. 178 (Oct, 2003 ), 1623-1629. 